\title{
Fixed and moving limit cycles for Liénard equations
}

\author{
Armengol Gasull ${ }^{1} \cdot$ Marco Sabatini ${ }^{2}$ \\ Received: 23 November 2018 / Accepted: 28 March 2019 / Published online: 9 April 2019 \\ (c) Fondazione Annali di Matematica Pura ed Applicata and Springer-Verlag GmbH Germany, part of Springer \\ Nature 2019
}

\begin{abstract}
We consider a family of planar vector fields that writes as a Liénard system in suitable coordinates. It has a fixed closed invariant curve that often contains periodic orbits of the system. We prove a general result that gives the hyperbolicity of these periodic orbits, and we also study the coexistence of them with other periodic orbits. Our family contains the celebrated Wilson polynomial Liénard equation, as well as all polynomial Liénard systems having hyperelliptic limit cycles. As an illustrative example, we study in more detail a natural 1-parametric extension of Wilson example. It has at least two limit cycles, one of them fixed and algebraic and the other one moving with the parameter, presents a transcritical bifurcation of limit cycles and for a given parameter has a non-hyperbolic double algebraic limit cycle. In order to prove that for some values of the parameter the system has exactly two hyperbolic limit cycles, we use several suitable Dulac functions.
\end{abstract}

Keywords Liénard equation · Limit cycle · Bifurcations · Invariant algebraic curve

Mathematics Subject Classification Primary 34C07; Secondary 37C23 · 34C25 · 37C27

\section{Introduction and main results}

Liénard equations

$$
\ddot{x}+f(x) \dot{x}+g(x)=0,
$$

are widely studied as models of physical, chemical, biological or electrical phenomena. A particular interesting case is when they contain algebraic limit cycles or polycycles. Wilson's paper [27] is maybe one of the first works dealing with this question. In the series of papers [2, $19,27,31]$, the study of hyperelliptic solutions $(y+R(x))^{2}+S(x)=0$ of polynomial Liénard

$\triangle$ Marco Sabatini

marco.sabatini@unitn.it

Armengol Gasull

gasull@mat.uab.cat

1 Dep. de Matemàtiques, Univ. Autònoma de Barcelona, 08193 Bellaterra, Barcelona, Catalonia, Spain

2 Dip. di Matematica, Univ. di Trento, 38123 Povo, TN, Italy 
equations is completed. The computations involved in these works are quite cumbersome, and some of them can be simplified by passing to some new coordinates in which the hyperelliptic solutions write as $y^{2}+S(x)=0$. In this work, we will consider the following family of smooth differential systems, which can be transformed into Eq. (1),

$$
\left\{\begin{array}{l}
\dot{x}=y-2 G(x) P(x), \\
\dot{y}=-G^{\prime}(x)(1+y P(x)),
\end{array}\right.
$$

with $G \in \mathcal{C}^{2}(\mathcal{I}, \mathbb{R}), P \in \mathcal{C}^{1}(\mathcal{I}, \mathbb{R})$, where $\mathcal{I} \subset \mathbb{R}$ is a given real interval. We denote by $X(x, y)=\left(X_{1}(x, y), X_{2}(x, y)\right)$ its associated vector field.

System (2) is equivalent to Liénard equation (1) with

$$
f(x)=3 G^{\prime}(x) P(x)+2 G(x) P^{\prime}(x) \text { and } g(x)=G^{\prime}(x)\left(1+2 G(x) P^{2}(x)\right) .
$$

Recall that given a smooth function $F(x, y)$ and a vector field $Y(x, y)=\left(Y_{1}(x, y)\right.$, $\left.Y_{2}(x, y)\right)$ such that

$$
\dot{F}(x, y)=\nabla F(x, y) \cdot Y(x, y)=K(x, y) F(x, y),
$$

for some smooth function $K$, then the curve $F(x, y)=0$ is invariant for the flow defined by $Y(x, y)$ and $K$ is called the cofactor of $F$. When $F, Y_{1}$ and $Y_{2}$ are polynomials, then $K$ is polynomial as well and its degree is at most the maximum degree of the components of $Y$ minus one.

As we will see, if we consider

$$
H(x, y)=y^{2}+2 G(x),
$$

the curve $H(x, y)=0$ is an invariant smooth curve for system (2). Moreover, when $\left[x^{-}, x^{+}\right] \subset \mathcal{I}$ is such that $G\left(x^{-}\right)=G\left(x^{+}\right)=0, G^{\prime}\left(x^{-}\right) G^{\prime}\left(x^{+}\right) \neq 0,\left.G\right|_{\left(x^{-}, x^{+}\right)}<0$ and $\left.\left(1+2 G(x) P^{2}(x)\right)\right|_{\left[x^{-}, x^{+}\right]}>0$, it holds that the oval

$$
\gamma:=\{H(x, y)=0\} \cap\left\{(x, y): x \in\left[x^{-}, x^{+}\right]\right\}
$$

is a periodic orbit of the system. In Proposition 6, we will show cases where $\gamma$ belongs to a continuum of periodic orbits. Next we give a set of generic hypotheses that, in the analytic case, will force that $\gamma$ is a limit cycle.

Non-degeneracy conditions (NDCs): Consider a periodic orbit $\gamma$ contained in the set $\{H(x, y)=0\}$ and also in the strip $\left\{(x, y): x \in\left[x^{-}, x^{+}\right]\right\}$where $\left[x^{-}, x^{+}\right] \subset \mathcal{I}$, $G\left(x^{-}\right)=G\left(x^{+}\right)=0, G^{\prime}\left(x^{-}\right) G^{\prime}\left(x^{+}\right) \neq 0$ and $\left.G\right|_{\left(x^{-}, x^{+}\right)}<0$. These NDCs are:

(a) If $\tilde{x} \in\left(x^{-}, x^{+}\right)$is such that $G^{\prime}(\tilde{x})=0$, then $G^{\prime \prime}(\tilde{x}) \neq 0$.

(b) The equation $G^{\prime}(x)=0$ has finitely many solutions in $\left(x^{-}, x^{+}\right)$. By hypothesis $(a)$, this number of solutions must be odd, $2 k-1,0<k \in \mathbb{N}$.

(c) When $k=1$, then $P^{\prime}(\tilde{x}) \neq 0$, where $\tilde{x}$ is the solution of $G^{\prime}(x)=0$ in $\left(x^{-}, x^{+}\right)$.

(d) When $k>1$, let $\tilde{x}_{1}, \tilde{x}_{2}, \ldots, \tilde{x}_{k-1}$ be the solutions of $G^{\prime}(x)=0$ in $\left(x^{-}, x^{+}\right)$such that $G^{\prime \prime}\left(\tilde{x}_{j}\right)<0$. Set

$$
\rho_{j}:=-\lambda_{j}^{+} / \lambda_{j}^{-}, \text {where } \lambda_{j}^{-}<0<\lambda_{j}^{+}
$$

are the eigenvalues of $D X$ at the critical points $\left(\tilde{x}_{j}, 2 G\left(\tilde{x}_{j}\right) P\left(\tilde{x}_{j}\right)\right)$, which are hyperbolic saddles. Then

$$
\Delta_{S}:=\prod_{k \in S} \rho_{k} \neq 1,
$$

for all non-empty subsets $S \subset\{1,2, \ldots, k-1\}$. 
As we will see, the above condition (d) is introduced to ensure that the polycycles that system (2) might have cannot be accumulation of periodic orbits (see Proposition 8).

Theorem 1 Let $\gamma$ be periodic orbit (4) of (2) contained in the strip $\left\{(x, y): x \in\left[x^{-}, x^{+}\right]\right\}$ where $\left[x^{-}, x^{+}\right] \subset \mathcal{I}, G\left(x^{-}\right)=G\left(x^{+}\right)=0, G^{\prime}\left(x^{-}\right) G^{\prime}\left(x^{+}\right) \neq 0$ and $\left.G\right|_{\left(x^{+}, x^{-}\right)}<0$. The following holds:

(i) When the non-degeneracy conditions (NDCs) hold and (2) is an analytic system, then $\gamma$ is a limit cycle.

(ii) When $\left.P^{\prime}\right|_{\left[x^{-}, x^{+}\right]}$does not change sign and vanish only on a set of measure zero, then $\gamma$ is a hyperbolic limit cycle, whose stability is given by the sign of $-P^{\prime}$ on $\left[x^{-}, x^{+}\right]$. Moreover, the system has not other limit cycles surrounded by $\gamma$.

In this paper, when we say that the stability of a limit cycle $\Gamma$ is given by the sign of some quantity $z, \operatorname{sgn}(z)$, we mean that when $z>0$ (resp. $z<0)$, the limit cycle $\Gamma$ is unstable (resp. stable).

Theorem 1 extends previous results of [1,17]. Item (i) was proved in [17] only in the case $P^{\prime}$ not zero in the whole interval. Item (ii) is totally new. In fact, the existence of limit cycles not contained in $H(x, y)=0$ has not been considered in that paper. The main tool for proving this result is the use of suitable Dulac functions.

Our second result studies in detail an extension of Wilson example, which we recall in Sect. 4.1. As we will see, its main interest is that it presents coexistence of a fixed limit cycle with other limit cycles and also a non-hyperbolic algebraic limit cycle. It is given by (2) with $G(x)=\left(x^{2}-1\right) / 2$ and $P(x)=x^{3}-b x$ and writes as

$$
\left\{\begin{array}{l}
\dot{x}=y-\left(x^{2}-1\right)\left(x^{3}-b x\right), \\
\dot{y}=-x\left(1+y\left(x^{3}-b x\right)\right) .
\end{array}\right.
$$

Such a system has the circle $y^{2}+2 G(x)=x^{2}+y^{2}-1=0$ as invariant algebraic curve. It is equivalent to Liénard equation (1) with $f(x)=9 x^{4}-5 b x^{2}-6 x^{2}+2 b$, and $g(x)=$ $x^{9}+(-1-2 b) x^{7}+b(2+b) x^{5}-b^{2} x^{3}+x$. We prove:

Theorem 2 Consider system (6) and the values $\underline{b} \simeq-1.44<b^{*} \simeq 0.747<\bar{b} \simeq 2.44$, given in Proposition 18. The following holds:

(i) It has the invariant algebraic curve $\gamma=\left\{x^{2}+y^{2}-1=0\right\}$ for all $b \in \mathbb{R}$. Moreover, $\gamma$ is a limit cycle if and only if $b \in(\underline{b}, \bar{b})$, and it is hyperbolic and stable if $b \in\left(\underline{b}, b^{*}\right)$ and hyperbolic and unstable if $b \in\left(b^{*}, \bar{b}\right)$. When $b=b^{*}$, the system has a transcritical bifurcation of limit cycles. In particular, when $b=b^{*}, \gamma$ is a double non-hyperbolic limit cycle, and when $b \gtrsim b^{*}$ (resp. $\left.b \lesssim b^{*}\right)$, a hyperbolic unstable (resp. stable) limit cycle bifurcates from $\gamma$ and it is surrounding (resp. surrounded by) $\gamma$.

(ii) It has no other limit cycle when $b \leq 0$.

(iii) When $0<b<b^{*}$, it has at least another limit cycle surrounded by $\gamma$. Moreover, when $0<b \leq 0.7$ it is unique, hyperbolic and unstable and the system has no limit cycle surrounding $\gamma$.

(iv) When $b>b^{*}$, it has at least another limit cycle surrounding $\gamma$. Moreover, when $b \geq$ 0.76 , it is unique, hyperbolic and stable and the system has no limit cycle surrounded by $\gamma$.

In fact, we believe that behavior of the limit cycles for system (6) is quite simple. The algebraic limit cycle $\gamma$ is fixed, and its position is independent of $b$. Then, varying $b$ a limit cycle $\Upsilon_{b}$ emerges for $b \gtrsim 0$ through a Hopf bifurcation, increases with $b$ and collides with $\gamma$ 
when $b=b^{*}$, interchanging their stabilities in a transcritical bifurcation of limit cycles, and then, it exists for all $b>b^{*}$. Although we have not been able to prove all the details of this description, all our results are compatible with this situation and we prove it for all values of $b$ outside a small neighborhood of $b^{*}$. In particular, it seems that the maximum number of limit cycles for system (6) is two, taking into account their multiplicities, and this upper bound is attained for $b \in(0, \bar{b})$. Nevertheless, more complicated behaviors, with more limit cycles, could be possible for $b \in(0.7,0.76)$.

The paper is organized as follows. Section 2 contains several preliminary results and tools that we will use along the work. Section 3 is devoted to prove Theorem 1. Finally, in Sect. 4 we apply Theorem 1 to several families of vector fields and we give the proof of Theorem 2 . One of the main tools for its proof is the use of suitable Dulac functions. In most cases, we reduce the problem of proving the uniqueness or the nonexistence of the limit cycles in a given region to the control of the sign of a polynomial function.

\section{Preliminary results}

This section contains several preliminary results about system (2). We study its critical points, the stability of its periodic orbits and the center problem. We also recall some general tools like the extended Bendixson-Dulac Theorem for controlling the number of limit cycles, a method for controlling the sign of one-parameter families of polynomials, the study of the stability of polycycles with hyperbolic corners and a result of Graef for general Liénard equations for knowing its behavior at infinity.

Lemma 3 System (2) has the invariant curve $H(x, y)=0$, with cofactor $K(x)=$ $-2 G^{\prime}(x) P(x)$. The critical points of system (2) are of two types:

(I) The intersections of the graph $y=2 G(x) P(x)$ with the lines $G^{\prime}(x)=0$;

(II) The intersections of the curve $1+y P(x)=0$ with the curve $H(x, y)=0$.

Proof The curve $H(x, y)=0$ is invariant since $\dot{H}(x, y)=-2 G^{\prime}(x) P(x) H(x, y)$. Moreover, one obtains the critical points of type (I) by equating to $0, X_{1}(x, y)$ and the first factor in $X_{2}(x, y)$.

The critical points of type (II) are obtained by observing that $1+y P(x)=0$ implies $y P(x) \neq 0$, hence $P(x)=-1 / y$ and

$$
0=X_{1}(x, y)=y-2 G(x) P(x)=y+\frac{2 G(x)}{y}=\frac{y^{2}+2 G(x)}{y}=\frac{H(x, y)}{y} ;
$$

hence, $H(x, y)$ has to vanish at such points.

Lemma 4 Set $\left[x^{-}, x^{+}\right] \subset \mathcal{I}$ such that $G\left(x^{-}\right)=G\left(x^{+}\right)=0, G^{\prime}\left(x^{-}\right) G^{\prime}\left(x^{+}\right) \neq 0$ and $\left.G\right|_{\left(x^{-}, x^{+}\right)}<0$. Then, if system (2) has no critical point of type (II) in the strip $\mathcal{S}=\{(x, y)$ : $\left.x \in\left[x^{-}, x^{+}\right]\right\}$, the oval

$$
\gamma=\{H(x, y)=0\} \cap \mathcal{S}
$$

is a periodic orbit of the system. Moreover, in this strip, all the critical points of type (I) are surrounded by $\gamma$.

Proof It is clear that the set $\gamma$ is an invariant oval for system (2). To prove that it is a periodic orbit, it suffices to see that the vector field $X$ has no critical point on $\gamma$. The hypothesis 
$G^{\prime}\left(x^{-}\right) G^{\prime}\left(x^{+}\right) \neq 0$ implies that $\left(x^{ \pm}, 0\right) \in \gamma$ are not critical points. Since we assume that there are not critical points of type (II), it holds that $1+2 G(x) P^{2}(x)>0$ for all $x \in\left[x^{-}, x^{+}\right]$. Take a critical point of type (I) in this interval, say $(\widetilde{x}, 2 G(\widetilde{x}) P(\tilde{x}))$. Then,

$$
H(\tilde{x}, 2 G(\tilde{x}) P(\tilde{x}))=4 G^{2}(\widetilde{x}) P^{2}(\tilde{x})+2 G(\widetilde{x})=2 G(\tilde{x})\left(1+2 G(\tilde{x}) P^{2}(\tilde{x})\right)<0 .
$$

Then, these critical points are surrounded by $\gamma$ as we wanted to prove.

It is well known that given a $T$-periodic orbit $\Gamma=\{x(t), y(t): t \in[0, T]\}$ of a $\mathcal{C}^{1}$ vector field $Y(x, y)$, then the sign of its characteristic exponent

$$
h(\Gamma)=\int_{0}^{T} \operatorname{div}(Y)(x(t), y(t)) \mathrm{d} t
$$

gives its stability, where $\operatorname{div}(X)(x, y)=\frac{\partial}{\partial x} Y_{1}(x, y)+\frac{\partial}{\partial y} Y_{2}(x, y)$ and $t$ denotes the time parametrization of $\Gamma$ (see, for instance, $[9,29,30])$. More, concretely if $h(\Gamma)>0($ resp. $<0)$, then $\Gamma$ is a hyperbolic unstable (resp. stable) limit cycle. When $h(\Gamma)=0$, the periodic orbit can be isolated and can belong to a continuum of periodic orbits or it can also be accumulation of infinitely many periodic orbits (isolated or not). Recall also that this latter case is not possible in the analytical setting.

In the particular case that $\Gamma \subset\{F(x, y)=0\}$, and the cofactor of $F$ is known, an alternative way of computing $h(\Gamma)$ is given in [14] (see also [13]). It holds that

$$
h(\Gamma)=\int_{0}^{T} K(x(t), y(t)) \mathrm{d} t .
$$

When the differential equation is written in polar coordinates, there is a third method to study the stability of a given periodic orbit (see Proposition 22).

By using (7) and (8), and ideas similar to those in [17], we will give some useful expressions to study the stability of the explicit periodic orbits of system (2).

Proposition 5 Let $\gamma$ be a periodic orbit of system (2) contained in $\{H(x, y)=0\}$. Then, it holds that

$$
h(\gamma)=4 \int_{0}^{T} G(x(t)) P^{\prime}(x(t)) \mathrm{d} t=-4 \int_{x^{-}}^{x^{+}} \frac{P^{\prime}(x) \sqrt{-2 G(x)}}{1+2 G(x) P^{2}(x)} \mathrm{d} x,
$$

where we use the same notation that in Lemma 4. In particular, if $h(\gamma) \neq 0$, then $\gamma$ is a hyperbolic limit cycle of (2).

Proof Notice that $\operatorname{div}(X)(x, y)=-3 G^{\prime}(x) P(x)-2 G(x) P^{\prime}(x)$. Moreover, by Lemma 3 the cofactor of the curve $H(x, y)=0$ is $K(x, y)=-2 G^{\prime}(x) P(x)$. Therefore by using (7) and (8), where

$$
\gamma=\{(x(t), y(t): t \in[0, T]\} \subset\{H(x, y)=0\},
$$

it holds that

$$
h(\gamma)=-\int_{0}^{T}\left(3 G^{\prime}(x(t)) P(x(t))+2 G(x(t)) P^{\prime}(x(t))\right) \mathrm{d} t=-\int_{0}^{T} 2 G^{\prime}(x(t)) P(x(t)) \mathrm{d} t
$$

and as a consequence

$$
\int_{0}^{T} G^{\prime}(x(t)) P(x(t)) \mathrm{d} t=-2 \int_{0}^{T} G(x(t)) P^{\prime}(x(t)) \mathrm{d} t .
$$


Hence,

$$
h(\gamma)=\int_{0}^{T} K(x(t)) \mathrm{d} t=-2 \int_{0}^{T} G^{\prime}(x(t)) P(x(t)) \mathrm{d} t=4 \int_{0}^{T} G(x(t)) P^{\prime}(x(t)) \mathrm{d} t .
$$

Let us parameterize the upper and lower parts of $\gamma$ as $\left(x, y^{ \pm}(x)\right)$ where $y^{ \pm}(x)=$ $\pm \sqrt{-2 G(x)}$. Then

$$
\begin{aligned}
h(\gamma) & =4 \int_{0}^{T} G(x(t)) P^{\prime}(x(t)) \mathrm{d} t \\
& =4 \int_{x^{-}}^{x^{+}} \frac{G(x) P^{\prime}(x)}{y^{+}(x)-2 G(x) P(x)} \mathrm{d} x+4 \int_{x^{+}}^{x^{-}} \frac{G(x) P^{\prime}(x)}{y^{-}(x)-2 G(x) P(x)} \mathrm{d} x \\
& =4 \int_{x^{-}}^{x^{+}} \frac{G(x) P^{\prime}(x)}{y^{+}(x)-2 G(x) P(x)} \mathrm{d} x+4 \int_{x^{-}}^{x^{+}} \frac{G(x) P^{\prime}(x)}{y^{+}(x)+2 G(x) P(x)} \mathrm{d} x \\
& =4 \int_{x^{-}}^{x^{+}} \frac{2 G(x) P^{\prime}(x) y^{+}(x)}{\left(y^{+}(x)\right)^{2}-4 G^{2}(x) P^{2}(x)} \mathrm{d} x=4 \int_{x^{-}}^{x^{+}} \frac{2 G(x) P^{\prime}(x) y^{+}(x)}{-2 G(x)-4 G^{2}(x) P^{2}(x)} \mathrm{d} x \\
& =-4 \int_{x^{-}}^{x^{+}} \frac{P^{\prime}(x) y^{+}(x)}{1+2 G(x) P^{2}(x)} \mathrm{d} x=-4 \int_{x^{-}}^{x^{+}} \frac{P^{\prime}(x) \sqrt{-2 G(x)}}{1+2 G(x) P^{2}(x)} \mathrm{d} x,
\end{aligned}
$$

as we wanted to prove.

By using the characterization of centers for Liénard equations given in $[3,6]$ in next result, we give a family of systems (2) for which an oval of $H(x, y)=0$ belongs to a continuum of periodic orbits surrounding a center.

Proposition 6 Consider an analytic system (2) and all the hypotheses and notation of Lemma 4. Let $\gamma$ be the periodic orbit of the system contained in $H(x, y)=0$ and in the strip $\mathcal{S}$. Assume also that $\mathbf{p}=(\tilde{x}, 2 G(\tilde{x}) P(\tilde{x}))$ is the unique critical point of (2) surrounded by $\gamma$ and that $G^{\prime \prime}(\tilde{x})>0$. Then, if $P(x)=\widehat{P}(G(x))$, for some analytic function $\widehat{P}$, the point $\mathbf{p}$ $i$ is a center and $\gamma$ belongs to the continuum of periodic orbits surrounding it.

Proof Notice that writing our system as a Liénard second-order equation following (3) we get that

$$
\begin{aligned}
& f(x)=3 G^{\prime}(x) P(x)+2 G(x) P^{\prime}(x)=\left(3 P(x)+2 G(x) \widehat{P}^{\prime}(G(x))\right) G^{\prime}(x) \\
& g(x)=G^{\prime}(x)\left(1+2 G(x) P^{2}(x)\right)=\left(1+2 G(x) \widehat{P}^{2}(G(x))\right) G^{\prime}(x) .
\end{aligned}
$$

Therefore, the primitives of both functions $f(x)$ and $g(x)$ are functions of $G(x)$, and moreover at $\tilde{x}, G^{\prime}(\tilde{x})=0$ and $G^{\prime \prime}(\tilde{x})>0$. These are the hypotheses of the results in $[3,6]$ that imply that system (2) has a center at p. By analyticity, the periodic orbits surrounding it include $\gamma$, as we wanted to prove.

Remark 7 (i) A particular and simple case covered by the previous proposition is $P(x) \equiv$ $p \in \mathbb{R} \backslash\{0\}$. In fact, it can be seen that in this case the function

$$
I(x, y)=\frac{1+2 p y-2 p^{2} G(x)}{(1+p y)^{2}}
$$

is a first integral of the system. Moreover, $H(x, y)=0$ coincides with the level set $I(x, y)=1$. 
(i) Under the hypotheses of previous proposition, it must hold that $h(\gamma)=0$. This can be seen directly by using Proposition 5 as follows:

$$
\begin{aligned}
h(\gamma) & =-4 \int_{x^{-}}^{x^{+}} \frac{P^{\prime}(x) \sqrt{-2 G(x)}}{1+2 G(x) P^{2}(x)} \mathrm{d} x=-4 \int_{x^{-}}^{x^{+}} \frac{\widehat{P}^{\prime}(G(x)) \sqrt{-2 G(x)} G^{\prime}(x)}{1+2 G(x) \widehat{P}^{2}(G(x))} \mathrm{d} x \\
& =\left.W(G(x))\right|_{x^{-}} ^{x^{+}}=W\left(G\left(x^{+}\right)\right)-W\left(G\left(x^{-}\right)\right)=0 .
\end{aligned}
$$

where $W(u)$ is a primitive of $-4 \widehat{P}^{\prime}(u) \sqrt{-2 u} /\left(1+2 u \widehat{P}^{2}(u)\right)$ in $u<0$.

Next we recall a very useful result for knowing the stability of some generic polycycles.

Proposition 8 [24] Let $G$ be a polycycle of an analytic vector field $Y$ with elementary saddles $\mathbf{p}_{1}, \mathbf{p}_{2}, \ldots, \mathbf{p}_{m}$ at its corners and respective eigenvalues $\lambda_{j}^{-}<0<\lambda_{j}^{+}, j=1,2, \ldots, m$. Then if $\Delta \doteq \prod_{j=1}^{m}-\lambda_{j}^{+} / \lambda_{j}^{-}$is less than 1 (resp. bigger than 1), then $G$ is an attracting (resp. repelling) polycycle.

To control the number of periodic orbits that are not included in $\{H(x, y)=0\}$, we will use the Bendixson-Dulac Theorem for multiple connected regions, $[5,12,20,28]$. To state it, recall that given an open subset $\mathcal{U}$ of $\mathbb{R}^{2}$ with smooth boundary, it is said to be $\ell$-connected if its fundamental group $\pi_{1}(\mathcal{U})$ is $\mathbb{Z} * \cdots * * \mathbb{Z}$, or in other words if the open set $\mathcal{U}$ has $\ell$ holes. Region with $\ell=0$ is also called simply connected. On them, the following theorem is the classical Dulac Theorem of nonexistence of periodic orbits.

Theorem 9 (Extended Bendixson-Dulac Theorem) Let $\mathcal{U}$ be an $\ell$-connected open subset of $\mathbb{R}^{2}$ with smooth boundary and let $X$ be a $\mathcal{C}^{1}$ vector field defined on it. Let $D: \mathcal{U} \rightarrow \mathbb{R}$ be a $\mathcal{C}^{1}$ function such that $M=\operatorname{div}(D X)$ does not change sign in $U$ and vanishes only on a null measure Lebesgue set, such that $\{M=0\} \cap\{D=0\}$ does not contain periodic orbits of $X$. Then, the maximum number of periodic orbits of this vector field totally contained in $\mathcal{U}$ is $\ell$. Furthermore, each one of them is a hyperbolic limit cycle that does not cut $\{D=0\}$ and its stability is given by the sign of DM over it.

To compute the function $M$ appearing in the above theorem, we will use several times the following lemma. Its proof is straightforward.

Lemma 10 Let $X$ be a $\mathcal{C}^{1}$ vector field, and let $F_{1}, F_{2}$ and $F_{3}$ be $\mathcal{C}^{1}$ functions, all defined in $\mathcal{U} \subset \mathbb{R}^{2}$. Assume that $F_{1}=0$ is a smooth invariant curve with cofactor $K$, that is, $Y\left(F_{1}\right)=K F_{1}$, and let $s_{1}, s_{2} \in \mathbb{R}$. Then

$$
\begin{aligned}
& \operatorname{div}\left(\left|F_{1}\right|^{s_{1}}\left|F_{2}\right|^{s_{2}} F_{3} X\right) \\
& =\operatorname{sgn}\left(F_{2}\right)\left|F_{1}\right|^{s_{1}}\left|F_{2}\right|^{s_{2}-1}\left(s_{1} K F_{2} F_{3}+s_{2} F_{3} X\left(F_{2}\right)+F_{2} X\left(F_{3}\right)+F_{2} F_{3} \operatorname{div}(X)\right) .
\end{aligned}
$$

Many times we will need to control the sign of a family of polynomials depending on a parameter. Next result is well known (see, for instance, [11]). As usual, we write $\triangle_{x}(p)$ to denote the discriminant of a polynomial $p(x)=a_{n} x^{n}+\cdots+a_{1} x+a_{0}$, that is,

$$
\triangle_{x}(p)=(-1)^{\frac{n(n-1)}{2}} \frac{1}{a_{n}} \operatorname{Res}\left(p(x), p^{\prime}(x)\right),
$$

where $\operatorname{Res}\left(p, p^{\prime}\right)$ is the resultant of $p$ and $p^{\prime}$, see [26]. 
Lemma 11 Let $p_{b}(x)=a_{n}(b) x^{n}+a_{n-1}(b) x^{n-1}+\cdots+a_{1}(b) x+a_{0}(b)$, be a family of real polynomials depending also polynomially on a real parameter $b$ and let $\Omega \subset \mathbb{R}$ be an open interval. Suppose that there exists another open interval $I=(u, v) \subset \mathbb{R}$ such that:

(i) There is some $b_{0} \in I$, such that $p_{b_{0}}(x)>0$ on $\Omega$;

(ii) For all $b \in I, \triangle_{x}\left(p_{b}(x)\right) \neq 0$;

(iii) For all $b \in I$, it holds that $a_{n}(b) \neq 0$ when $(u, v)=\mathbb{R}$, or that $p_{b}(u) p_{b}(v) \neq 0$ when $u, v \in \mathbb{R}$.

Then for all $b \in I, p_{b}(x)>0$ on $\Omega$.

We end this section with a result of Graef for general continuous Liénard equation that allows to know the behavior of the solutions at infinity. It is said that the solutions of a Liénard equation (1) are uniformly ultimately bounded if there exists a constant $K>0$ such that for any solution $x(t)$ of (1) there is a time $\tau$ such that for all $t>\tau$ we have $|x(t)|<K$ and $|\dot{x}(t)|<K$.

Theorem 12 [18, Thm. 3.1] Define $F(x)=\int_{0}^{x} F(s) \mathrm{d} s$ and assume that there exist positive constants $k$ and $c$ such that

(i) $x F(x)>0$ and $x g(x)>0$ if $|x| \geq k$;

(ii) $F(x) \geq c>0$ if $x \geq k$ or $F(x) \leq-c<0$ if $x \leq-k$.

Then

$$
\int_{0}^{ \pm \infty}(f(x)+|g(x)|) \mathrm{d} x= \pm \infty
$$

is a sufficient condition for all solutions of (1) to be uniformly ultimately bounded.

The stability of the infinity for polynomial Liénard differential equations (1) can also be studied by using the tools introduced in [8]. Notice that system (6) writes as (1) with $f$ and $g$ the polynomials given in (3).

\section{Proof of Theorem 1}

To prove (i), we start by studying the nature of the critical points of $X$ surrounded by $\gamma$. Let $\mathbf{p}=(\tilde{x}, 2 G(\tilde{x}) P(\tilde{x}))$, with $G^{\prime}(\tilde{x})=0$, one of them. The differential matrix $D X$ at this point is

$$
D X(\mathbf{p})=\left(\begin{array}{cc}
-2 G(\tilde{x}) P^{\prime}(\tilde{x}) & 1 \\
-G^{\prime \prime}(\tilde{x})\left(1+2 G(\tilde{x}) P^{2}(\tilde{x})\right) & 0
\end{array}\right) .
$$

By Lemma 3, since $\gamma$ is a periodic orbit, $\left.\left(1+2 G(x) P^{2}(x)\right)\right|_{x \in\left[x^{-}, x^{+}\right]}>0$. Moreover, by NDC (a), it holds that $G^{\prime \prime}(\tilde{x}) \neq 0$. Then $\operatorname{sgn}\left(\operatorname{det}(D X(\mathbf{p}))=\operatorname{sgn}\left(G^{\prime \prime}(\tilde{x})\right)\right.$. Therefore, the minima of $G$ correspond to points of index +1 , which are hyperbolic node or focus when $P^{\prime}(\tilde{x}) \neq 0$ and weak focus when $P^{\prime}(\tilde{x})=0$. Similarly, the maxima of $G$ are hyperbolic saddle points.

To show that $\gamma$ is a limit cycle we argue by contradiction. Assume that it is not. Then, since system (2) is analytic, it holds that $\gamma$ belongs to a continuum of periodic orbits contained in $\{H(x, y) \leq 0\} \cap\left\{(x, y): x^{-} \leq x \leq x^{+}\right\}$. By considering this continuum, and again by the analiticity of $X$ and the Poincaré-Bendixson theory, we know that this continuum of periodic orbits must have a critical point $\mathbf{q}$ in its interior boundary $B$. 
If $B=\{\mathbf{q}\}$, then $k=1$ in the notation introduced when we give the non-degeneracy conditions. Therefore, $\mathbf{q}=(\tilde{x}, 2 G(\tilde{x}) P(\tilde{x}))$ must be a center, implying that $P^{\prime}(\tilde{x})=0$ fact that is in contradiction with the NDC (c).

If $B \neq\{\mathbf{q}\}$, then the continuum of periodic orbits ends in a polycycle whose corners must be some of the $k-1$ hyperbolic saddles that $X$ has in the region surrounded by $\gamma$. The NDC (d) prevents this fact, because the set of conditions (5) implies by Proposition 8 that all possible polycycles are either attracting or repelling polycycles. Thus we have again a contradiction, and (i) follows.

(ii) We will apply the extended Bendixson-Dulac Theorem to $X$ in the simply connected region $\mathcal{U}$ given by the bounded region surrounded by $\gamma$. Thus, $\ell=0$ in Theorem 9 . We take $F_{1}=H, F_{2}=F_{3}=1$ and $s_{1}=s$ in Lemma 10 . Then, $K=-2 G^{\prime} P$ and

$$
\begin{aligned}
\operatorname{div}\left(|H|^{s} X\right) & =|H|^{s}(s K+\operatorname{div}(X))=-|H|^{s}\left(2 s G^{\prime} P+3 G^{\prime} P+2 G P^{\prime}\right) \\
& =-|H|^{s}\left((2 s+3) G^{\prime} P+2 G P^{\prime}\right) .
\end{aligned}
$$

Then, $\operatorname{div}\left(|H|^{-3 / 2} X\right)=-2|H|^{-3 / 2} G P^{\prime}$ does not change sign and vanishes only of a set of measure zero of vertical straight lines. Therefore, system (3) has no limit cycle in $\mathcal{U}$ as we wanted to prove. That $\gamma$ is hyperbolic and that its stability is given by the sign of $-P^{\prime}$ follows from the expression of $h(\gamma)$ proved in Proposition 5.

\section{Examples and proof of Theorem 2}

In this section, we study in more detail several particular examples of system (2). We consider Wilson equation, equations with many periodic orbits or many hyperbolic limit cycles and an extension of Wilson equation that presents a fixed limit cycle together with a moving limit cycle, as well as a non-hyperbolic algebraic limit cycle.

\subsection{Wilson example and systems with hyperelliptic limit cycles}

Wilson in [27] gives the first example of Liénard equation with an explicit algebraic hyperelliptic limit cycle $\left(y+\mu x\left(x^{2}-4\right) / 4\right)^{2}+x^{2}-4=0$ for $|\mu|<2$. Wilson example writes as

$$
\ddot{x}+\mu\left(x^{2}-1\right) \dot{x}+\mu^{2} x^{3}\left(x^{2}-4\right) / 16+x=0 .
$$

It is easy to see that if we take $G(x)=x^{2} / 2-c$ and $P(x)=b x$ in (2), we get

$$
\left\{\begin{array}{l}
\dot{x}=y-b x\left(x^{2}-2 c\right), \\
\dot{y}=-x(1+b x y),
\end{array}\right.
$$

and Wilson example corresponds to $c=2$ and $b=\mu / 4$. Notice that Wilson example is a cubic system when we write it as in (2) and it is of degree five written as an usual Liénard system.

As a first corollary of our results, we get the following proposition.

Proposition 13 System (10) has limit cycles if and only if $0<|b|<1 / c$ and $c>0$. When these inequalities hold, the limit cycle is unique, algebraic, hyperbolic and it is the ellipse $y^{2}+2 G(x)=y^{2}+x^{2}-2 c=0$. Moreover, its stability is given by the sign of $-b$. 
Proof By Lemma 3, the conditions on the parameter for $\gamma$ to be a periodic orbit are precisely $c>0$ and $0<|b|<1 / c$. By changing $x \rightarrow-x$ and $t \rightarrow-t$ if $b<0$, we can reduce this case to the case $b>0$. Moreover, by Theorem 1 , since $P^{\prime}(x) G(x)=\left(x^{2} / 2-c\right) b$ does not change sign on the strip where $\gamma$ lies, then $\gamma$ is hyperbolic and stable, because its stability is given by the $-\operatorname{sgn}\left(P^{\prime}\right)=-\operatorname{sgn}(b)<0$. Furthermore, we know that the system has no limit cycle surrounded by $\gamma$.

To study the nonexistence of limit cycles in the exterior of $\gamma$, we will apply again the extended Poincaré-Dulac Theorem (Theorem 9) to $X$ in this region. In fact, we consider the two regions $\mathcal{U}_{1}$ and $\mathcal{U}_{2}$ given by the bounded and unbounded regions delimited by $\gamma$, respectively, because this approach allows to treat simultaneously both regions. Thus, $\ell=0$ for $\mathcal{U}_{1}$ and $\ell=1$ for $\mathcal{U}_{2}$ in Theorem 9. We take $F_{1}=H, F_{2}=F_{3}=1$ and $s_{1}=s$ in Lemma 10 , and we get

$$
\begin{aligned}
\operatorname{div}\left(|H|^{s} X\right) & =-|H|^{s}\left(2 s G^{\prime} P+3 G^{\prime} P+2 G P^{\prime}\right) \\
& =-b|H|^{s}\left((2 s+3) x^{2}+x^{2}-2 c\right)=-b|H|^{s}\left((2 s+4) x^{2}-2 c\right) .
\end{aligned}
$$

Then, taking $s=-2$, we get $\operatorname{div}\left(|H|^{-2} X\right)=2 b c|H|^{-2}$ which does not vanish on $\mathcal{U}_{j}, j=$ 1,2 . Therefore, we have proved again that system (3) has no limit cycle in the interior of $\gamma$. Moreover, we get that it has at most one limit cycle in its exterior. Furthermore, if it exists, it is hyperbolic and its stability is given by the $\operatorname{sgn}(b c)=\operatorname{sgn}(b)>0$, so it would be repeller. This is in contradiction with the uniqueness of this periodic orbit and Theorem 12, which implies that infinity of system (10) with $b>0$ is also a repeller, because when $\gamma$ is a periodic orbit, the origin is the unique critical point of the system.

When $\gamma$ is not a periodic orbit, then it contains all the critical points of the system different from the origin, and we can argue as in the previous paragraph, proving also that the system has no periodic orbits outside $\gamma$. Then in this situation system (10) has not periodic orbits.

For Wilson example, Proposition 13 can also be obtained from the results of [10] where it is proved that it has a Darboux first integral.

Remark 14 The results for system (10) can be extended to other $G$ with qualitative behavior like to the one of $G(x)=x^{2} / 2-c$. For instance, taking $P(x)=b x$ and $G(x)=x^{2 n} /(2 n)-$ $c, n \in \mathbb{N}$ a similar result to Proposition 13 holds. To prove it, it suffices to follow the same steps that in the study of $(10)$ and then take $s=-(3 n+1) /(2 n)$ when we apply Lemma 10 and Theorem 9.

\subsubsection{Systems with hyperelliptic limit cycles}

In papers [19,31], polynomial Liénard equations with hyperelliptic limit cycles are written as

$$
\ddot{x}+\left(R^{\prime}(x)+\frac{1}{2} R(x) \frac{Q^{\prime}(x)}{Q(x)}\right) \dot{x}-\frac{1}{2} Q^{\prime}(x)+\frac{1}{2} R^{2}(x) \frac{Q^{\prime}(x)}{Q(x)}=0,
$$

for suitable polynomials $R$ and $Q$. We remark that taking in our system $P=-R / Q$ and $G(x)=-Q(x) / 2$ we get Eq. (11).

We also remark that in order to have a polynomial system (2) the possibility of choosing $G$ and $P$ polynomial is not the only one. If we take $G$ polynomial and $W$ is any factor of $\operatorname{gcd}\left(G(x), G^{\prime}(x)\right)$, then taking $P(x)=\tilde{P}(x) / W(x)$ with $\tilde{P}$ also polynomial gives a polynomial system (2). 


\subsection{Systems with many periodic orbits}

We start this section with systems having many centers.

Proposition 15 (i) There exist polynomial systems of form (2) of degree $2 k$, having $k$ nonnested centers.

(ii) There exist analytic systems of form (2) having infinitely many non-nested centers.

Proof The proofs are straightforward consequences of Proposition 6 and Remark 7. (i) Consider system (2) with $P(x) \equiv 1$ and $G(x)=\prod_{j=1}^{k}(x-j)^{2}$. Then, the degree of the system is $2 k$. Moreover, following the proof of (i) of Theorem 1 we get that the critical points $(j, 0)$, $j=1,2, \ldots, k$ are weak foci. By Remark 7 , the system has the smooth first integral $I(x, y)$ given in (9), well defined in a neighborhood of all these $k$ points. Hence, all them are centers.

For proving (ii), we can consider $G(x)=\sin ^{2}(x)$ and again $P(x) \equiv 1$. The proof is the same, and all points $(j \pi, 0), j \in \mathbb{Z}$ are centers.

A similar result to Proposition 15 but for limit cycles instead of centers is the following:

Proposition 16 (i) There exist polynomial systems of form (2) of degree $2 k+1$, having $k$ hyperbolic non-nested limit cycles.

(ii) There exist analytic systems of form (2) having infinitely many hyperbolic non-nested limit cycles.

Proof (i) Consider

$$
G(x)=\prod_{j=1}^{k}(x-j)^{2}-c \text { and } P(x)=b x
$$

in system (2). Take $c>0$ fixed, such that $G(x)$ has $2 k$ simple zeroes and $0<b$ small enough. By using (ii) of Theorem 1, we can easily prove that the curve $H(x, y)=0$ has exactly $k$ non-nested ovals. All them are periodic orbits because the equation $1+y P(x)=$ 0 writes as $x y=-1 / b$, and for $0<b$ small enough, this hyperbola does not cut these ovals. Moreover, again by Theorem 1, they are hyperbolic and attractive limit cycles and the system has not more limit cycles inside them.

(ii) Consider $G(x)=\cos (x)$ and $P(x)=-1 /\left(4+x^{2}\right)$. Then, it is easy to see that we are under the hypotheses of (ii) of Theorem 1 and the set $H(x, y)=y^{2}+\cos x=0$ contains infinitely many non-nested hyperbolic limit cycles and each of them does not surround other limit cycles.

Remark 17 Notice that when we write the polynomial systems given in item (i) of Propositions 15 and 16 as Liénard equations (1), they have degrees $4 k-1$ and $4 k+1$, respectively.

\subsection{A degree five family and proof of Theorem 2}

In this section, we will study the number of limit cycles of system (6). For the sake of clarity, we write it again

$$
\left\{\begin{array}{l}
\dot{x}=y-\left(x^{2}-1\right)\left(x^{3}-b x\right), \\
\dot{y}=-x\left(1+y\left(x^{3}-b x\right)\right) .
\end{array}\right.
$$

Recall that it has the circle $y^{2}+2 G(x)=x^{2}+y^{2}-1=0$ as invariant algebraic curve. 


\subsubsection{The existence and stability of the algebraic limit cycle}

Proposition 18 System (2) has the periodic orbit $\gamma$, of equation $H(x, y)=x^{2}+y^{2}-1=0$, if and only if $b \in(\underline{b}, \bar{b})$, where $b<0<\bar{b}$ are the only real roots of the polynomial $R(b)=$ $4 b^{6}-12 b^{5}-4 b^{4}+28 b^{3}+56 b^{2}-72 b-229$. Moreover, there exists $b^{*} \in(0.746,0.749)$ such that $\gamma$ is:

(i) a hyperbolic stable limit cycle when $b \in\left(\underline{b}, b^{*}\right)$;

(ii) a non-hyperbolic limit cycle when $b=b^{*}$;

(iii) a hyperbolic unstable limit cycle when $b \in\left(b^{*}, \bar{b}\right)$.

Numerically, $b^{*} \simeq 0.747024$. Moreover, $\underline{b}$ and $\bar{b}$ can be expressed by radicals, $\bar{b} \simeq 2.440096$ and $\underline{b}=1-\bar{b} \simeq-1.440096$.

Proof By Lemma 3, we already know that $\gamma$ is an invariant curve. In order to prove that $\gamma$ is a periodic orbit, it is sufficient to prove that (6) has no critical point in $\gamma$. By Lemma 3, this is equivalent to show that the polynomial

$$
\begin{aligned}
S_{b}(x) & =1+2 G(x) P^{2}(x)=1+x^{2}\left(x^{2}-b\right)^{2}\left(x^{2}-1\right) \\
& =x^{8}-(2 b+1) x^{6}+(b+2) b x^{4}-b^{2} x^{2}+1
\end{aligned}
$$

has no real root in the interval $[-1,1]$. By using Sturm method and computing the discriminant of $S_{b}(x)$, we obtain precisely the condition of the statement, because $\triangle_{x}\left(S_{b}\right)=256 R^{2}(b)$. For more details about the use of Sturm sequences, see [25].

The polynomial $R$ can be written as $R(b)=-q(b(1-b))$, where $q(z)=4 z^{3}+16 z^{2}+$ $72 z+229=0$. Hence, its zeros can be found by radicals. In particular, $\underline{b}$ and $\bar{b}$ are the solutions of $b(1-b)=z_{0}$, where $z_{0}$ is the real root of $q(z)$, which writes as

$$
z_{0}=-\frac{A}{6}+\frac{76}{3 A}-\frac{4}{3}, \text { where } A=\sqrt[3]{4103+273 \sqrt{273}} .
$$

Take now $\gamma$ for $b \in(\underline{b}, \bar{b})$. By Proposition 5 its stability is given by

$$
r(b) \doteq h(\gamma)=-4 \int_{-1}^{1} \frac{P^{\prime}(x) \sqrt{-2 G(x)}}{1+2 G(x) P^{2}(x)} \mathrm{d} x=\int_{0}^{1} U_{b}(x) \sqrt{1-x^{2}} \mathrm{~d} x .
$$

where $U_{b}$ is the rational function $U_{b}(x)=8\left(b-3 x^{2}\right) / S_{b}(x)$.

Although we cannot compute (13) in terms of elementary functions, there is a procedure that will allow us to get $r(b)$ with any desired precision.

Before starting to study more properties of $r(b)$, first notice that for $b \in(\underline{b}, \bar{b})$, it holds that $S_{b}(x)>0$. Moreover, for $b \leq 0, U_{b}(x) \leq 0$ and so for all $b \leq 0, r(b)<0$ giving that $\gamma$ is a hyperbolic stable limit cycle, as we wanted to prove.

From now on, we will assume that $b \in(0, \bar{b})$. Notice that

$$
r^{\prime}(b)=\int_{0}^{1} \frac{\partial}{\partial b} U_{b}(x) \sqrt{1-x^{2}} \mathrm{~d} x .
$$

After some computations, we get that

$$
\frac{\partial}{\partial b} U_{b}(x)=\frac{-8\left(5 x^{8}-(6 b+5) x^{6}+b(b+6) x^{4}-b^{2} x^{2}-1\right)}{S_{b}^{2}(x)}
$$

and using the same tools as before, we get that $\frac{\partial}{\partial b} U_{b}(x)>0$; hence, $r^{\prime}(b)>0$. Moreover, we know that $r(0)<0$ and $\lim _{b \rightarrow \bar{b}} r(b)=+\infty$, because $S_{\bar{b}}(x)$ vanishes in $(0,1)$. Therefore, 
there exists a unique value of $b$, say $b=b^{*}$, such that $r\left(b^{*}\right)=0$. For such a value, $\gamma$ is no longer hyperbolic.

Let us prove that $b^{*} \in(0.746,0.749)$. For the sake of conciseness, we will give the full details for proving that $b^{*}<0.8$, while for the rest of the proof we only show the main differences with this easier bound.

We want to prove that $r(0.8)>0$, that is, $\int_{0}^{1} U_{0.8}(x) \sqrt{1-x^{2}}>0$. We will bound inferiorly $U_{0.8}(x)$ by a polynomial $p(x)$ in $\mathbb{Q}[x]$, such that $U_{0.8}(x)>p(x)$ for $x \in[0,1]$, and such that

$$
\int_{0}^{1} U_{0.8}(x) \sqrt{1-x^{2}}>\int_{0}^{1} p(x) \sqrt{1-x^{2}}>0 .
$$

Notice that then the right-hand inequality can be analytically proved because for any $n \in \mathbb{N}$,

$$
\int_{0}^{1} x^{n} \sqrt{1-x^{2}}=\int_{0}^{\pi / 2} \sin ^{n} \theta \cos ^{2} \theta \mathrm{d} \theta=I_{n}-I_{n+2},
$$

where

$$
I_{n}=\int_{0}^{\pi / 2} \sin ^{n} \theta \mathrm{d} \theta= \begin{cases}\frac{(2 m-1) ! !}{(2 m) ! !} \frac{\pi}{2}, & \text { if } n=2 m, \\ \frac{(2 m-2) ! !}{(2 m-1) ! !}, & \text { if } n=2 m-1,\end{cases}
$$

with $0<m \in \mathbb{N}, 0 ! !=1 ! !=1$ and for $2 \leq k \in \mathbb{N}, k ! !=k \times(k-2) ! !$.

In order to find a suitable polynomials $p$, we fix a degree (in this case 3 ) and we compute the interpolating polynomial for $U_{0.8}(x)-\eta$, for some positive $\eta$. First we check numerically for a good $\eta$, selecting $\eta=0.2$. Then, we get a degree 3 polynomial with big rational coefficients that interpolates the points of Table 1 .

Afterward, by hand we tune a little bit the values of Table 1, proposing as interpolation values the ones of Table 2, where the numbers of the second row are approximations of the numbers of Table 1 . This new interpolating polynomial corresponding to the new Table 2 looks easier. It is $p(x)=\left(27 x^{3}-279 x^{2}+12 x+62\right) / 10$. Hence

By using once more Sturm method, we can prove that for $x \in[0,1], U_{0.8}(x)>p(x)$.

$$
r(0.8)=\int_{0}^{1} U_{0.8}(x) \sqrt{1-x^{2}} \mathrm{~d} x>\int_{0}^{1} p(x) \sqrt{1-x^{2}} \mathrm{~d} x=\frac{19}{25}-\frac{31}{160} \pi>0 .
$$

In order to prove that $r(0.749)>0$, we use as new $p(x)$ the polynomial of degree 8 corresponding to the interpolating polynomial of the table

$$
\left(j / 8, U_{0.749}(j / 8)-1 / 200\right), \quad j=0,1, \ldots, 8 .
$$

Table 1 Some values of $U_{0.8}(x)-\eta$, with $\eta=0.2$

Table 2 Final table for finding $p(x)$

\begin{tabular}{lllll}
\hline$x$ & 0 & $\frac{1}{3}$ & $\frac{2}{3}$ & 1 \\
\hline$U_{0.8}(x)-\frac{1}{5}$ & $\frac{31}{5}$ & $\frac{2905463}{781685} \simeq 3.7$ & $-\frac{731621}{158905} \simeq-4.6$ & $-\frac{89}{5}$ \\
\hline
\end{tabular}

\begin{tabular}{lllll}
\hline$x$ & 0 & $\frac{1}{3}$ & $\frac{2}{3}$ & 1 \\
\hline$p(x)$ & $\frac{31}{5}$ & $\frac{18}{5}$ & $-\frac{23}{5}$ & $-\frac{89}{5}$ \\
\hline
\end{tabular}


Similarly, in order to prove that $h_{0.746}(\gamma)<0$, we use another polynomial of degree 8 , this time corresponding to the interpolating polynomial of the table

$$
\left(j / 8, U_{0.746}(j / 8)+1 / 300\right), \quad j=0,1, \ldots, 8 .
$$

Now, it can be proved that this new polynomial $q$ is an upper bound of $U_{0.746}$, and it holds that

$$
r(0.746)=\int_{0}^{1} U_{0.746}(x) \sqrt{1-x^{2}} \mathrm{~d} x<\int_{0}^{1} q(x) \sqrt{1-x^{2}} \mathrm{~d} x<0 .
$$

The fact that when $b=b^{*}, \gamma$ is a limit cycle and is a consequence of statement (i) of Theorem 1 because in this case the origin is the only critical point of the system and it is not a center.

\subsubsection{The system in polar coordinates and other useful properties}

The expression of (6) in polar coordinates is

$$
\left\{\begin{array}{l}
\dot{r}=r\left(r^{2}-1\right)\left(b-r^{2} \cos ^{2} \theta\right) \cos ^{2} \theta \\
\dot{\theta}=-1+b \sin \theta \cos \theta-r^{2} \sin \theta \cos ^{3} \theta .
\end{array}\right.
$$

By using the above expression, we get the following results.

Lemma 19 (i) For $b \leq 0$, the only periodic orbit of system (6) is $x^{2}+y^{2}=1$ and it exists if and only if $b \in(\underline{b}, 0]$.

(ii) Apart of $r=1$, system (6) for $b>0$ has not other periodic orbits in the region $\{r \leq \sqrt{b}\}$.

Proof Item (i) follows because when $b \leq 0$ then $\dot{r}\left(r^{2}-1\right) \leq 0$. To prove item (ii), notice that when $b>0$, then

$$
\left.\dot{r}\right|_{r=\sqrt{b}}=\sqrt{b}(b-1) b \sin ^{2} \theta \cos ^{2} \theta,
$$

and moreover, when $r \leq \sqrt{b}, \dot{r}\left(r^{2}-1\right) \geq 0$.

The existence for system (6) of a limit cycle different of $\gamma=\left\{x^{2}+y^{2}=1\right\}$ when $b \neq b^{*}$ is guaranteed by the following results.

Lemma 20 System (6) has a Hopf bifurcation at the origin when $b=0$. More specifically, an unstable periodic orbit bifurcates from the origin when $b \gtrsim 0$. Moreover, for $0<b<b^{*}$ the system has at least one periodic orbit in $\mathcal{U}_{1} \doteq\left\{x^{2}+y^{2}<1\right\}$.

Proof The existence of a Hopf bifurcation follows from straightforward computations. Moreover, a limit cycle always exists in $\mathcal{U}_{1}$ because of the Poincaré-Bendixson theory. For these values of $b$, both the origin and $\gamma$ are attractors (see (i) in Proposition 18) and the origin is the only critical point of the system.

Lemma 21 For $b>b^{*}$, system (6) has at least one periodic orbit in $\mathcal{U}_{2} \doteq\left\{x^{2}+y^{2}>1\right\}$.

Proof We start proving that the set $x^{2}+y^{2}=1$, which recall is invariant for the flow of system (6), is a repeller for $b \geq b^{*}$. We split the study in two cases $b \in\left(b^{*}, \bar{b}\right)$ and $b \in[\bar{b}, \infty)$.

In the first case, $b \in\left(b^{*}, \bar{b}\right)$, by item (ii) in Proposition $18, \gamma=\left\{x^{2}+y^{2}=1\right\}$ is a hyperbolic unstable limit cycle. 
When $b \in[\bar{b}, \infty)$, although the set $x^{2}+y^{2}-1=0$ is invariant, it is no more a periodic orbit. Let us prove that it contains two couples of critical points (resp. one couple of double critical points) when $b>\bar{b}$ (resp. $b=\bar{b}$ ) and four (resp. two) regular orbits. Moreover, these critical points are two hyperbolic saddles and two hyperbolic nodes (resp. two semihyperbolic saddle nodes). It is convenient to choose polar expression (14) of the vector field $X$. Call $Y$, its associated vector field. On $r=1$, the critical points are $(r, \theta)=\left(1, \theta^{*}\right)=\mathbf{p}$, where $\theta^{*}$ are the solutions of

$$
W_{b}(\theta)=-1+b \sin \theta \cos \theta-\sin \theta \cos ^{3} \theta=0
$$

and

$$
D Y(\mathbf{p})=\left(\begin{array}{cc}
2\left(b-\cos ^{2} \theta^{*}\right) \cos ^{2} \theta^{*} & 0 \\
-2 \sin \theta^{*} \cos ^{3} \theta^{*} & W_{b}^{\prime}\left(\theta^{*}\right)
\end{array}\right) .
$$

Notice that the number of solutions of (15) varies as the number of solutions of $S_{b}(x)=0$ (see (12)). For $b>\bar{b}$ (resp. $b=\bar{b}$ ), both equations have four simple (resp. two double) solutions.

In any case, the eigenvalues of the critical points corresponding to the normal direction to $r=1$ are $\lambda\left(\theta^{*}\right) \doteq 2\left(b-\cos ^{2} \theta^{*}\right) \cos ^{2} \theta^{*}>0$ and the other ones, corresponding to the tangent direction to the invariant set $r=1$, are $W_{b}^{\prime}\left(\theta^{*}\right)$. Hence, the only critical points on $r=1$ are two couples of hyperbolic repeller nodes and two couples of hyperbolic saddles (resp. a couple of semi-hyperbolic saddle nodes) when $b>\bar{b}$ (resp. $b=\bar{b}$ ), as we wanted to prove. Moreover, because for all $\theta^{*}, \lambda\left(\theta^{*}\right)>0$, we get that the set $x^{2}+y^{2}-1=0$ is repeller.

Moreover, we can prove that for all $b \in \mathbb{R}$ system (6) is under the hypotheses of Theorem 12 ; hence, all the solutions are attracted to a compact set. In particular, infinity is a repeller. Hence, by using the Poincaré-Bendixson theory and the fact that the system has no critical points in $\mathcal{U}_{2}$, the existence of a limit cycle in $\mathcal{U}_{2}$ follows.

When $b \in(\underline{b}, \bar{b})$, and in a neighborhood of $r=1$, system (14) is equivalent to

$$
\frac{\partial r}{\partial \theta}=S(r, \theta)=\frac{-r\left(r^{2}-1\right)\left(b-r^{2} \cos ^{2} \theta\right) \cos ^{2} \theta}{1-b \sin \theta \cos \theta+r^{2} \sin \theta \cos ^{3} \theta} .
$$

By using next result of Lloyd, we will prove the existence of a transcritical bifurcation of limit cycles at $\gamma$ when $b=b^{*}$. In particular, we will prove that when $b=b^{*}, \gamma$ is a double limit cycle. As usual, given a $\mathcal{C}^{2}$, non-autonomous $2 \pi$-periodic differential equation $\frac{d r}{d \theta}=T(r, \theta)$, we denote by $R(\theta, \rho)$ the solution such that $R(0, \rho)=\rho$ and by $\Pi(\rho) \doteq R(2 \pi, \rho)$ its Poincaré map. In particular, the fixed points of the Poincaré map, which correspond to the zeroes of the displacement map $\Delta(\rho) \doteq \Pi(\rho)-\rho$, are initial conditions that give rise to periodic orbits of the differential equation.

Proposition 22 [20] Consider a $\mathcal{C}^{2}$ non-autonomous $2 \pi$-periodic differential equation $\frac{d r}{d \theta}=$ $T(r, \theta)$ and let $\Pi$ be its Poincaré return map. Then

$$
\begin{aligned}
\Pi^{\prime}(\rho) & =\exp \left(\int_{0}^{2 \pi} \frac{\partial T(R(\theta, \rho), \theta)}{\partial r} \mathrm{~d} \theta\right) \\
\Pi^{\prime \prime}(\rho) & =\Pi^{\prime}(\rho)\left[\int_{0}^{2 \pi} \frac{\partial^{2} T(R(\theta, \rho), \theta)}{\partial r^{2}} \exp \left(\int_{0}^{\theta} \frac{\partial T(R(\psi, \rho), \psi)}{\partial r} \mathrm{~d} \psi\right) d \theta\right] .
\end{aligned}
$$

Proposition 23 When $b=b^{*}$, system (6) has a transcritical bifurcation of limit cycles at $\gamma=\{r=1\}$. In particular, when $b=b^{*}, \gamma$ is a double non-hyperbolic limit cycle, and when 
$b \gtrsim b^{*}$ (resp. $\left.b \lesssim b^{*}\right)$, a hyperbolic unstable (resp. stable) limit cycle bifurcates from $\gamma$ and it is surrounding (resp. surrounded by) $\gamma$.

Proof To prove this result, we use polar expression (16) of (6), which recall that it is valid in a neighborhood of $b=b^{*}$ and $r=1$. We also remark that all results about the stability of the periodic orbits for Eq. (16) must be reversed when we deal with system (6), because $\dot{\theta}<0$ in a neighborhood of $r=1$, and when $t$ increases, $\theta$ decreases, and vice versa.

By Proposition 22, the stability of $r=1$ for (16) is given by the sign of

$$
\left.\widehat{r}(b) \doteq \int_{0}^{2 \pi} \frac{\partial S(r, \theta)}{\partial r}\right|_{r=1} \mathrm{~d} \theta=\int_{0}^{2 \pi} \frac{2\left(\cos ^{2} \theta-b\right) \cos ^{2} \theta}{1-b \sin \theta \cos \theta+\sin \theta \cos ^{3} \theta} \mathrm{d} \theta,
$$

In fact, it can be seen that $\widehat{r}(b)=-r(b)$, where $r(b)$ is given in (13) (as we have explained the minus sign comes from the fact that $\dot{\theta}<0$ on $r=1$ ).

Formula (17) or its equivalent rational expression

$$
\widehat{r}(b)=4 \int_{-\infty}^{\infty} \frac{1-b-b u^{2}}{\left(u^{4}-b u^{3}+2 u^{2}+(1-b) u+1\right)\left(1+u^{2}\right)} \mathrm{d} u,
$$

seems worst than (13) for having good estimates of $b^{*}$. Anyway, formula (17) provides a simple proof that $r^{\prime}(b)>0$, fact used in the proof of Proposition 18, because

$$
\widehat{r}^{\prime}(b)=\int_{0}^{2 \pi} \frac{-2 \cos ^{2} \theta}{\left(1-b \sin \theta \cos \theta+\sin \theta \cos ^{3} \theta\right)^{2}} \mathrm{~d} \theta<0 .
$$

If we call $\Delta(\rho, b)$ the displacement map for (16), where notice that we have added its dependence on $b$, we know that $\Delta(1, b) \equiv 0$. In particular, $\frac{\partial \Delta(1, b)}{\partial b} \equiv \frac{\partial^{2} \Delta(1, b)}{\partial b^{2}} \equiv 0$. Moreover, from Proposition 22 and (18),

$$
\frac{\partial \Delta(1, b)}{\partial \rho}=e^{\widehat{r}(b)}-1 \quad \text { and } \quad K(b) \doteq \frac{\partial^{2} \Delta(1, b)}{\partial \rho \partial b}=e^{\widehat{r}(b)} \widehat{r}^{\prime}(b)<0 .
$$

We claim that $L:=\frac{\partial^{2} \Delta\left(1, b^{*}\right)}{\partial \rho^{2}}>0$. Then we are under the hypotheses of the Weierstrass preparation Theorem and we get that

$$
\begin{aligned}
\Delta(\rho, b)= & \Delta\left(1, b^{*}\right)+\frac{\partial \Delta\left(1, b^{*}\right)}{\partial \rho}(\rho-1)+\frac{\partial \Delta\left(1, b^{*}\right)}{\partial b}\left(b-b^{*}\right)+\frac{1}{2} \frac{\partial^{2} \Delta\left(1, b^{*}\right)}{\partial \rho^{2}}(\rho-1)^{2} \\
& +\frac{\partial^{2} \Delta\left(1, b^{*}\right)}{\partial \rho \partial b}(\rho-1)\left(b-b^{*}\right)+\frac{1}{2} \frac{\partial^{2} \Delta\left(1, b^{*}\right)}{\partial b^{2}}(b-1)^{2}+O_{3}\left(\rho-1, b-b^{*}\right) \\
= & \frac{L}{2}(\rho-1)^{2}+K\left(b^{*}\right)(\rho-1)\left(b-b^{*}\right)+O_{3}\left(\rho-1, b-b^{*}\right) .
\end{aligned}
$$

In particular, equation $\Delta(\rho, b)=0$ has only two solutions in a neighborhood of $(\rho, b)=$ $\left(1, b^{*}\right)$. They are $\rho=1$ and

$$
\rho=1-\frac{2 \widehat{r}^{\prime}\left(b^{*}\right)}{L}\left(b-b^{*}\right)+O_{2}\left(b-b^{*}\right),
$$

which are the initial conditions of the periodic orbits of (16) near $r=1$. Hence for $b \lesssim b^{*}$ a hyperbolic stable limit cycle, contained in $\mathcal{U}_{1}$ tends to $r=1$ when $b \rightarrow b^{*}$ and crosses it giving rise to a hyperbolic unstable limit cycle, contained in a neighborhood of $r=1$ in $\mathcal{U}_{2}$. This proves the lemma.

To end the proof we need to prove the claim. Notice that it is equivalent to show that $r=1$ is a double limit cycle. 
By Proposition 22, it suffices to prove that

$$
L=\left.\int_{0}^{2 \pi} \frac{\partial^{2} S(r, \theta)}{\partial r^{2}}\right|_{r=1, b=b^{*}} \exp \left(\left.\int_{0}^{\theta} \frac{\partial S(r, \psi)}{\partial r}\right|_{r=1,=b^{*}} d \psi\right) \mathrm{d} \theta>0
$$

We write $L=\int_{0}^{2 \pi} \frac{A_{b^{*}}(\theta) C_{b^{*}}(\theta)}{B_{b^{*}}(\theta)} \mathrm{d} \theta$ where

$$
\begin{aligned}
A_{b}(\theta) \doteq & 6 b^{2} \cos ^{3} \theta \sin \theta-6 b\left(2 \cos ^{3} \theta \sin \theta+1\right) \cos ^{2} \theta+2 \cos ^{4} \theta\left(3 \cos ^{3} \theta \sin \theta+7\right), \\
B_{b}(\theta) \doteq & b^{2} \sin ^{2} \theta \cos ^{2} \theta+2 b \cos \theta\left(\cos ^{5} \theta-\cos ^{3} \theta-\sin \theta\right) \\
& -\cos ^{8} \theta+\cos ^{6} \theta+2 \cos ^{3} \theta \sin \theta+1, \\
C_{b}(\theta) \doteq & \exp \left(\int_{0}^{\theta} \frac{2\left(\cos ^{2} \psi-b\right) \cos ^{2} \psi}{1-b \sin \psi \cos \psi+\sin \psi \cos ^{3} \psi} \mathrm{d} \psi\right) .
\end{aligned}
$$

We do not know the exact value of $b^{*}$, but by Proposition 18 we know that $b^{*} \in \mathcal{J} \doteq$ $\left(b_{\ell}, b_{u}\right)=(0.746,0.749)$. It holds that for $b \in \mathcal{J}$,

$$
A_{b}(\theta)>T(\theta), \quad \frac{3}{5}<\frac{1}{B_{b}(\theta)}<\frac{9}{5} \text { and } \frac{3}{5}<C_{b}(\theta)<\frac{7}{5},
$$

where $T$ is the piecewise linear $\pi$-periodic map

$$
T(\theta)= \begin{cases}8-32 \theta / \pi, & 0 \leq \theta<\pi / 4 \\ -1 / 2, & \pi / 4 \leq \theta<3 \pi / 4 \\ 32 \theta / \pi-24, & 3 \pi / 4 \leq \theta<\pi\end{cases}
$$

Then

$$
\begin{aligned}
L & >2\left(\int_{0}^{\pi / 4}\left(8-\frac{32 \theta}{\pi}\right) \frac{3}{5} \frac{3}{5} \mathrm{~d} \theta-\int_{\pi / 4}^{3 \pi / 4} \frac{1}{2} \frac{9}{5} \frac{7}{5} \mathrm{~d} \theta+\int_{3 \pi / 4}^{\pi}\left(\frac{32 \theta}{\pi}-24\right) \frac{3}{5} \frac{3}{5} \mathrm{~d} \theta\right) \\
& =\frac{9 \pi}{50}>0 .
\end{aligned}
$$

The proof of each of the inequalities given in (19) needs some work. As an example, we will give the full details to show that for $b \in \mathcal{J}$ and $\theta \in(\pi / 4,3 \pi / 4), A_{b}(\theta)>T(\theta) \equiv-1 / 2$.

First notice that studying the sign of the coefficients in $b$ of $A_{b}(\theta)$ we get that

$$
A_{b}(\theta)> \begin{cases}\mathcal{A}_{1}(\theta), & \theta \in(\pi / 4, \pi / 2), \\ \mathcal{A}_{2}(\theta), & \theta \in(\pi / 2,3 \pi / 4),\end{cases}
$$

where

$$
\begin{aligned}
& \mathcal{A}_{1}(\theta)=6 b_{\ell}^{2} \cos ^{3} \theta \sin \theta-6 b_{u}\left(2 \cos ^{3} \theta \sin \theta+1\right) \cos ^{2} \theta+2 \cos ^{4} \theta\left(3 \cos ^{3} \theta \sin \theta+7\right), \\
& \mathcal{A}_{2}(\theta)=6 b_{u}^{2} \cos ^{3} \theta \sin \theta-6 b_{u}\left(2 \cos ^{3} \theta \sin \theta+1\right) \cos ^{2} \theta+2 \cos ^{4} \theta\left(3 \cos ^{3} \theta \sin \theta+7\right) .
\end{aligned}
$$

To prove that $\mathcal{A}_{1}(\theta)+1 / 2>0$ for $\theta \in(\pi / 2,3 \pi / 4)$, it is convenient to use the rational parametrization of $x^{2}+y^{2}=1$. Then $\sin \theta=2 r /\left(1+r^{2}\right)$ and $\cos \theta=\left(1-r^{2}\right) /\left(1+r^{2}\right)$, and the interval $\theta \in(\pi / 2,3 \pi / 4)$ is transformed into $r \in(\sqrt{2} / 2-1,1)$ and

$$
\left.\left(\mathcal{A}_{1}(\theta)+\frac{1}{2}\right)\right|_{\sin \theta=2 r /\left(1+r^{2}\right), \cos \theta=\left(1-r^{2}\right) /\left(1+r^{2}\right)}=\frac{1}{62500} \frac{P_{16}(r)}{\left(r^{2}+1\right)^{8}},
$$


where $P_{16}(r)=625375 r^{16}-43887 r^{15}-\cdots+43887 r+625375$ is a polynomial without real roots and positive. This fact is proved by using the Sturm method. Hence $\mathcal{A}_{1}(\theta)+\frac{1}{2}>0$, as we wanted to prove. The function $\mathcal{A}_{2}(\theta)+\frac{1}{2}$ in $(\pi / 2,3 \pi / 4)$ can be studied in a similar way. The proof that $A_{b}(\theta)>T(\theta)$, in the other intervals, can be done using the same change of the independent variable. The only difference is that it also appears the function $\theta=\arctan \left(2 r /\left(1-r^{2}\right)\right)$, but it can be bounded by a polynomial map, arriving at the end to a polynomial condition that can be treated again by using the Sturm method.

Lemma 24 Let $\mathcal{U}_{1}$ and $\mathcal{U}_{2}$ the bounded and unbounded regions of $\mathbb{R}^{2} \backslash \gamma$, respectively. Then, system (6) is a semi-complete family of rotated vector fields on both invariant regions $U_{j}, j=1,2$.

Proof Following [7,21-23], it suffices to compute

$$
X_{1} \frac{\partial X_{2}}{\partial b}-X_{2} \frac{\partial X_{1}}{\partial b}=x^{2}\left(x^{2}+y^{2}-1\right)
$$

and to observe that the above quantity does not change sign on each $U_{j}$, and vanishes only on the straight line $x=0$, which is not invariant by the flow of the system.

The fact that system (6) is a semi-complete family of rotated vector fields allows to know the movement of the periodic orbits contained in each $U_{j}$ when $b$ varies. One of the most useful properties is the so called non-intersection property. Fixed one $\mathcal{U}_{j}$, it asserts that if $\Upsilon_{1}$ and $\Upsilon_{2}$ are limit cycles corresponding to systems (6) with different values of $b$, then $\Upsilon_{1} \cap \Upsilon_{2}=\emptyset$. Informally, it says that if for a set of values of $b$ some limit cycles cover a region of the phase plane, this region turns out to be forbidden for the periodic orbits that the system could have for others values of the parameter. Another nice property in $\mathcal{U}_{1}$ (resp. $\mathcal{U}_{2}$ ) is that stable limit cycles (resp. unstable limit cycles) increase when the parameter $b$ also increases. As a consequence, the study of the bifurcation diagrams for such families is simpler.

\subsubsection{The second limit cycle}

Recall that $\mathcal{U}_{1}=\left\{x^{2}+y^{2}<1\right\}$ and $\mathcal{U}_{2}=\left\{x^{2}+y^{2}>1\right\}$. In this section, we will study the number of limit cycles of system (6) on each of these regions, according to the values of $b>0$, by applying again the extended Poincaré-Dulac Theorem (Theorem 9) to this system. Recall that the case $b \leq 0$ is already solved in Lemma 19 .

The first results deals with the easier case $b \geq 1$. Recall that we already know by Lemma 19 that the system has no limit cycle in $\mathcal{U}_{1}$. This approach treats together both regions $\mathcal{U}_{j}, j=$ 1,2 .

Proposition 25 Consider system (6) with $b \geq 1$. The following holds:

(i) It has no periodic orbit in $\mathcal{U}_{1}$.

(ii) It has exactly one periodic orbit in $\mathcal{U}_{2}$, which is a hyperbolic stable limit cycle.

Proof We apply the extended Poincaré-Dulac Theorem to $X$ in both regions $\mathcal{U}_{j}, j=1,2$. Notice that $\ell=0$ for $\mathcal{U}_{1}$ and $\ell=1$ for $\mathcal{U}_{2}$ in the theorem. We take once more $F_{1}=H, F_{2}=$ $F_{3}=1$ and $s_{1}=s$ in Lemma 10 and we get

$$
\begin{aligned}
\operatorname{div}\left(|H|^{s} X\right) & =|H|^{s}\left(-2 s G^{\prime} P-3 G^{\prime} P-2 G P^{\prime}\right) \\
& =|H|^{s}\left(-(2 s+6) x^{4}+(2 b s+4 b+3) x^{2}-b\right) .
\end{aligned}
$$


We introduce $N_{b}(s, u)=-(2 s+6) u^{2}+(2 b s+4 b+3) u-b$. Notice that the discriminant with respect to $u$ of $N_{b}(s, u)$ is $A_{b}(s) \doteq 4 b^{2} s^{2}+\left(16 b^{2}+4 b\right) s+16 b^{2}+9$. Therefore, when $b \geq 1$, we can take the solution of $A_{b}(s)=0$,

$$
s^{+}=\frac{-1-4 b+2 \sqrt{2(b-1)}}{2 b} .
$$

It can be seen that $2 s^{+}+6 \neq 0$. Then, $A_{b}\left(s^{+}\right) \equiv 0$ and the function $N_{b}\left(s^{+}, u\right) \leq 0$ because it has a double zero. As a consequence,

$$
\operatorname{div}\left(|H|^{s^{+}} X\right)=|H|^{s^{+}} N_{b}\left(s^{+}, x^{2}\right) \leq 0 .
$$

By Theorem 9, we have proved again that when $b \geq 1$, system (6) has no limit cycle in $\mathcal{U}_{1}$, and moreover, that it has at most one limit cycles in $\mathcal{U}_{2}$. Moreover, when it exists, it is hyperbolic and stable.

In fact, by Lemma 21 we get that for $b \geq 1$, system (6) has exactly one limit cycle in $\mathcal{U}_{2}$, which is hyperbolic and stable.

It remains to study the case $b \in(0,1)$. We get complete results outside a small neighborhood of $b=b^{*}$, the value for which $\gamma$ is an algebraic non-hyperbolic limit cycle. We divide the study in two propositions. The first one covers $b \in[0.76,1)$, while the second one studies $b \in(0,0.70]$.

Proposition 26 Consider system (6) with $b \in[0.76,1)$. The following holds:

(i) It has no periodic orbit in $\mathcal{U}_{1}$.

(ii) It has exactly one periodic orbit in $\mathcal{U}_{2}$, which is a hyperbolic stable limit cycle.

Proof We will use Bendixson-Dulac Theorem in $\mathcal{U}_{j}, j=1,2$. To apply Lemma 10, we choose $F_{1}(x, y)=H(x, y)=x^{2}+y^{2}-1, s_{1}=-5 / 2, F_{2}=1$ and $F_{3}(x, y)=y^{2 n}+$ $\sum_{j=0}^{2 n-1} c_{j}(x) y^{j}$ is a polynomial to be determined. The value $s_{1}=-5 / 2$ is selected after several trials. Then

$$
\operatorname{div}\left(|H|^{-5 / 2} F_{3} X\right)=|H|^{-5 / 2}\left(-5 K F_{3} / 2+X\left(F_{3}\right)+F_{3} \operatorname{div}(X)\right) \doteq|H|^{-5 / 2} D_{n},
$$

where recall that $K(x)=-x^{2}\left(x^{2}-b\right)$ is the cofactor of $H=0$. By using the same tools that in the study of Liénard systems in its usual expression (see $[4,15,16]$ ), it can be seen that, fixed $n \in \mathbb{N}$, we can choose in a unique way the polynomials $c_{j}(x)$ in such a manner that $c_{j}(0)=0$ and $D_{n}(x, y)$ is a function that depends only of $x$. The reason is that this condition forces simple linear differential equations for each $c_{j}$, with triangular structure, starting with $c_{n-1}(x)$ and ending with $c_{0}(x)$. As an example, we detail the computations for $n=2$.

The function $D_{2}$ writes as

$$
\begin{aligned}
D_{2}(x, y)= & \left(c_{1}^{\prime}(x)-b+(3+b) x^{2}-3 x^{4}\right) y^{2}+\left(\left(-b x+(b+1) x^{3}-x^{5}\right) c_{1}^{\prime}(x)\right. \\
& \left.+\left(-b+3 x^{2}-2 x^{4}\right) c_{1}(x)+c_{0}^{\prime}(x)-2 x\right) y \\
& +\left(\left(-b x+(b+1) x^{3}-x^{5}\right) c_{0}^{\prime}(x)+\left(-b+(3-b) x^{2}-x^{4}\right) c_{0}(x)\right. \\
& \left.+c_{0}^{\prime}(x)-c_{1}(x) x\right)
\end{aligned}
$$


Then, it is clear that there are unique polynomials satisfying $c_{0}(0)=c_{1}(0)=0$ such that $D_{2}$ depends only on $x$. Choosing these polynomials, we get that $D_{2}(x, y)=x^{2} d_{2, b}(x)$, where

$$
\begin{aligned}
d_{2, b}(x)= & -\frac{231}{50} x^{12}+\left(\frac{903 b}{100}+\frac{3297}{200}\right) x^{10}-\left(\frac{21 b^{2}}{4}+\frac{6629 b}{200}+\frac{819}{40}\right) x^{8} \\
& +\left(\frac{5 b^{3}}{6}+\frac{58 b^{2}}{3}+\frac{1737 b}{40}+9\right) x^{6}-\left(\frac{35 b^{3}}{12}+\frac{1597 b^{2}}{60}+21 b+\frac{18}{5}\right) x^{4} \\
& +\left(\frac{47 b^{3}}{12}+\frac{4 b}{3}+15 b^{2}+6\right) x^{2}-b\left(3 b^{2}+4\right) .
\end{aligned}
$$

We will apply Lemma 11 to $\Omega=\mathbb{R}$ to prove that for $b \in I=\left(b_{2}, 1\right)$ where $b_{2} \simeq 0.906$ is the real root of $t(b)=350 b^{3}-740 b^{2}+1307 b-837=0$, it holds that $d_{2, b}(x) \neq 0$.

In fact, $t(b)$ is a factor of $\triangle_{x}\left(d_{2, b}\right)$, which is a polynomial of degree 51 in $\mathbb{Q}[b]$. By using Sturm algorithm, it can be seen that $b_{2}$ is the smallest positive root of $\triangle_{x}\left(d_{2, b}\right)$. Hence, hypothesis (ii) of the lemma follows. Moreover, the coefficient of $x^{12}$ of $d_{b}$ is $-231 / 50$, so it is never zero and hypothesis (iii) of the lemma also is satisfied. Finally, using once more Sturm algorithm we can prove that $d_{2,0.95}(x) \neq 0$, and as a consequence, hypothesis (i) is satisfied for $0.95=b_{0} \in I$. Then, $d_{b}(x) \neq 0$ for $x \in \mathbb{R}$ and $b \in I$, as wanted to prove.

Then by the Bendixson-Dulac Theorem, for $b \in\left(b_{2}, 1\right)$, the system has no limit cycle in $\mathcal{U}_{1}$ and at most one limit cycle, hyperbolic and stable whenever it exists, in $\mathcal{U}_{2}$. Moreover, this limit cycle always exists by Lemma 21.

To show that the proposition holds, we proceed as in the case $n=2$ but taking $n=60$. Then after many computations, done in Maple 18 , we get the same result for $b \in\left(b_{60}, 1\right)$ where $b_{60} \simeq 0.7595$ is the smallest positive real root of the corresponding $\triangle_{x}\left(d_{60, b}\right)$.

Although theoretically it is possible to increase $n$ in the proof of previous proposition to have a bigger interval for which Proposition 26 holds, the complexity and time needed to perform all the computations make us to stop at $n=60$.

Proposition 27 Consider system (6) with $b \in(0,0.7]$. The following holds:

(i) It has exactly one periodic orbit in $\mathcal{U}_{1}$, which is a hyperbolic unstable limit cycle.

(ii) It has no periodic orbit in $\mathcal{U}_{2}$.

Proof We will apply again Bendixson-Dulac Theorem, but in the $\mathcal{U}_{1} \backslash\{(0,0)\}$, that has $\ell=1$, for proving the uniqueness of limit cycle in $\mathcal{U}_{1}$. Here, to apply Lemma 10 we choose $F_{1}(x, y)=H(x, y)=x^{2}+y^{2}-1, s_{1}=-2 ; F_{2}(x, y)=x^{2}+y^{2}, s_{2}=-13 / 4$ and $F_{3}(x, y)=y^{4}+\sum_{j=0}^{3} c_{j}(x) y^{j}$ is a polynomial to be determined. In this case, higher degrees of $F_{3}$ seem not to increase the interval of applicability of Bendixson-Dulac Theorem. The values $s_{1}$ and $s_{2}$ are chosen by hand, after experimenting a little bit with the functions. In this situation $\operatorname{div}\left(|H|^{-2} F_{2}^{-13 / 4} F_{3} X\right)=|H|^{-2} F_{2}^{-17 / 4} D$, where

$$
D=-2 K F_{2} F_{3}-13 F_{3} X\left(F_{2}\right) / 4+F_{2} X\left(F_{3}\right)+F_{2} F_{3} \operatorname{div}(X) .
$$

Similarly that in the previous proposition, we can prove that there is a unique choice of $c_{j}(x), j=0,1,2,3$, such that all $c_{j}(0)=0$ and

$$
D(x, y)=D_{b}(x, y)=\alpha_{2}(x, b) y^{2}+\alpha_{1}(x, b) y+\alpha_{0}(x, b),
$$

for some polynomials $\alpha_{j}(x, b)$. To force that $D_{b}$ does not change sign on $\mathcal{U}_{1}$ we impose that

$$
E_{b}(x) \doteq \alpha_{1}^{2}(x, b)-4 \alpha_{0}(x, b) \alpha_{1}(x, b)<0 \quad \text { in } \quad x \in[-1,1] \backslash\{0\} .
$$


By using Lemma 11 for $x \in I:=(-1,1)$ and $b \in(0,0.7)$, the result follows. In this occasion, the applicability of the lemma arrives until $\widehat{b} \simeq 0.7024$, a real root of $\triangle_{x}\left(E_{b}(x) / x^{10}\right)$.

If we impose that $E_{b}(x)<0$ for all $x \in \mathbb{R} \backslash\{0\}$, we get that this holds for $b$ between $b^{\prime} \simeq 0.4068$, another root of $\triangle_{x}\left(E_{b}(x) / x^{10}\right)$ and $\widehat{b}$. This implies the nonexistence of limit cycles in $\mathcal{U}_{2}$ because, by using the Bendixson-Dulac Theorem, in this region we would have at most one (hyperbolic) limit cycle, and this is in contradiction with the fact that the stabilities of infinity and $r=1$ are reversed.

To cover the interval $b \in(0,0.41]$, we repeat the computations, but now taking $F_{1}(x, y)=$ $H(x, y)=x^{2}+y^{2}-1, s_{1}=s_{2}=-5 / 2, F_{2}(x, y)=x^{2}+y^{2}-b^{2}$, and again a polynomial $F_{3}(x, y)=y^{4}+\sum_{j=0}^{3} c_{j}(x) y^{j}$ satisfying the above restrictions, but changing the initial condition $c_{2}(0)=0$ by $c_{2}(0)=1$. We can ensure that the possible limit cycles do not cut the curve $F_{2}=0$ because in the proof of the Lemma 19 we show that for $0<b<1$ it is crossed inwards by the flow. We obtain that the new $E_{b}$ is negative for all $x \in \mathbb{R} \backslash\{0\}$, for $b$ between 0 and $0.447 \ldots$, a root of $\triangle_{x}\left(E_{b}(x) / x^{4}\right)$. Then the result follows arguing as in the previous situation.

Proof of Theorem 2 Item (i) is a consequence of Propositions 18 and 23. Item (ii) follows from Lemma 19. Lemma 20 and Proposition 27 imply item (iii). Finally, item (iv) follows from Lemma 21 and Proposition 25 when $b \geq 1$ and from Lemma 21 and Proposition 26 when $b \in[0.76,1)$.

Acknowledgements The first author is partially supported by the MINECO/FEDER MTM2016-77278-P and AGAUR 2017-SGR-1617 Grants. The second author is partially supported by GNAMPA, Gruppo Nazionale per l'Analisi Matematica, la Probabilità e le loro Applicazioni.

\section{References}

1. Abdelkader, M.A.: Relaxation oscillators with exact limit cycles. J. Math. Anal. Appl. 218, 308-312 (1998)

2. Chavarriga, J., García, I.A., Llibre, J., Zoladek, H.: Invariant algebraic curves for the cubic Liénard system with linear damping. Bull. Sci. Math. 130, 428-441 (2006)

3. Cherkas, L.A.: Conditions for a Liénard equation to have a centre. Differ. Equ. 12, 201-206 (1976)

4. Cherkas, L.A.: Estimation of the number of limit cycles of autonomous systems. Differ. Equ. 13, 529-547 (1977)

5. Cherkas, L.A.: Dulac function for polynomial autonomous systems on a plane. Differ. Equ. 33, 692-701 (1997)

6. Christopher, C.J., Lloyd, N.G., Pearson, J.M.: On a Cherkass method for centre conditions. Nonlinear World 2, 459-469 (1995)

7. Duff, G.F.D.: Limit-cycles and rotated vector fields. Ann. Math. 57, 15-31 (1953)

8. Dumortier, F., Herssens, C.: Polynomial Liénard equations near infinity. J. Differ. Equ. 153, 1-29 (1999)

9. Dumortier, F., Llibre, J., Artés, J.C.: Qualitative Theory of Planar Differential Systems. Universitext. Springer, Berlin (2006)

10. García, I.A., Giacomini, H., Giné, J.: Generalized nonlinear superposition principles for polynomial planar vector fields. J. Lie Theory 15, 89-104 (2005)

11. García-Saldaña, J.D., Gasull, A., Giacomini, H.: Bifurcation values for a familiy of planar vector fields of degree five. Discrete Contin. Dyn. Syst. 35, 669-701 (2015)

12. Gasull, A., Giacomini, H.: Some applications of the extended Bendixson-Dulac theorem. In: Ibáñez, S., Pérez del Río, J., Pumariño, A., Rodríguez, J. (eds.) Progress and Challenges in Dynamical Systems, pp. 233-252. Springer, Berlin (2013)

13. Gasull, A., Giné, J., Grau, M.: Multiplicity of limit cycles and analytic m-solutions for planar differential systems. J. Differ. Equ. 240, 375-398 (2007)

14. Giacomini, H., Grau, M.: On the stability of limit cycles for planar differential systems. J. Differ. Equ. 213, 368-388 (2005) 
15. Giacomini, H., Neukirch, S.: Number of limit cycles of the Liénard equation. Phys. Rev. E 56, 3809-3813 (1997)

16. Giacomini, H., Neukirch, S.: Algebraic approximations to bifurcation curves of limit cycles for the Liénard equation. Phys. Lett. A 244, 53-58 (1998)

17. Giné, J., Grau, M.: A note on: "Relaxation oscillators with exact limit cycles". J. Math. Anal. Appl. 324, 739-745 (2006)

18. Graef, J.R.: On the generalized Liénard equation with negative damping. J. Differ. Equ. 12, 34-62 (1972)

19. Liu, C., Chen, G., Yang, J.: On the hyperelliptic limit cycles of Liénard systems. Nonlinearity 25, 16011611 (2012)

20. Lloyd, N.G.: A note on the number of limit cycles in certain two-dimensional systems. J. Lond. Math. Soc. 20, 277-286 (1979)

21. Perko, L.M.: Global families of limit cycles of planar analytic systems. Trans. Am. Math. Soc. 322, 627-656 (1990)

22. Perko, L.M.: Rotated vector fields. J. Differ. Equ. 103, 127-145 (1993)

23. Perko, L.M.: Differential Equations and Dynamical Systems. Texts in Applied Mathematics, vol. 7, Third edn. Springer, New York (2001)

24. Sotomayor, J.: Curvas definidas por Equaçoes Diferenciais no Plano. Instituto de Matemática Pura e Aplicada, Rio de Janeiro (1981)

25. Stoer, J., Bulirsch, R.: Introduction to Numerical Analysis. Springer, New York (1980)

26. Sturmfels, B.: Solving Systems of Polynomial Equations, CBMS Regional Conference Series Mathematics, vol. 97. American Mathematical Society, Providence, RI (2002) (Published for the Conference Board of the Mathematical Sciences, Washington, DC)

27. Wilson, J.C.: Algebraic periodic solutions of $\ddot{x}+f(x) \dot{x}+g(x)=0$. Contrib. Differ. Equ. 3, 1-20 (1964)

28. Yamato, K.: An effective method of counting the number of limit cycles. Nagoya Math. J. 76, 35-114 (1979)

29. Yan-Qian, Y., et al.: Theory of Limit Cycles. Translations of Mathematical Monographs, vol. 66. American Mathematical Society, Providence, RI (1986)

30. Zhang, Z.F., et al.: Qualitative theory of differential equations. Translations of Mathematical Monographs, vol. 101. American Mathematical Society, Providence, RI (1992)

31. Zoladek, H.: Algebraic invariant curves for the Liénard equation. Trans. Am. Math. Soc. 350, 1681-1701 (1998)

Publisher's Note Springer Nature remains neutral with regard to jurisdictional claims in published maps and institutional affiliations. 